\title{
Impact of an Antimicrobial Stewardship Program Comprehensive Care Bundle on Management of Candidemia
}

Allen Antworth, Curtis D. Collins, Anjly Kunapuli, Kristin Klein, Peggy Carver, Tejal Gandhi, Laraine Washer, and Jerod L. Nagel

Study Objective. To analyze the impact of a comprehensive care bundle directed by an antimicrobial stewardship team (AST) on the management of candidemia.

Design. Single-center, quasi-experimental study.

Setting. A 930-bed academic hospital.

Patients. Seventy-eight patients with candidemia were evaluated; 41 patients received the candidemia care bundle (AST group), and 37 did not (historical control group).

Measurements and Main Results. A candidemia care bundle was developed by an interdisciplinary AST, incorporating key elements from the Infectious Diseases Society of America's Clinical Practice Guidelines for the Management of Candidemia. The AST made prospective recommendations in accordance with the care bundle. Bundle elements were utilization of appropriate antifungal agents with appropriate duration of use, removal of intravenous catheters, repeat blood cultures, monitoring of time until clearance of candidemia, and performance of ophthalmologic examinations. Compliance with all candidemia care bundle elements was significantly higher in the AST group versus the control group ( $78.0 \%$ vs $40.5 \%, \mathrm{p}=0.0016$ ). Implementation of the care bundle significantly improved rates of ophthalmologic examination ( $97.6 \%$ vs $75.7 \%, \mathrm{p}=0.0108)$, selection of appropriate antifungal therapy ( $100 \%$ vs $86.5 \%, \mathrm{p}=0.0488$ ), and compliance with an appropriate duration of therapy $(97.6 \%$ vs $67.7 \%, \mathrm{p}=0.0012)$. In addition, the AST group had fewer excess total days of therapy beyond the recommended duration than the control group ( 5 vs 83 total antifungal days). Length of hospitalization ( 20 vs 21 days, $\mathrm{p}=0.9184$ ), time until clearance of candidemia ( 3 vs 3 days $\mathrm{p}=0.610$ ), rate of persistent candidemia ( $22 \%$ vs $40.5 \%, \mathrm{p}=0.126$ ), and rate of recurrent candidemia ( $4.9 \%$ vs $5.4 \%, \mathrm{p}=0.916$ ) were similar in the AST group versus the control group.

Conclusion. A comprehensive candidemia care bundle directed by our institution's AST improved the management of patients with candidemia. We encourage further exploration into the use of care bundles by ASTs as part of their multifaceted approach to promoting appropriate antimicrobial utilization and optimizing the management of patients with infectious diseases.

Key Words: candidemia, candida, care bundle, stewardship.

(Pharmacotherapy 2013;33(2):137-143) 
The ultimate goal of antimicrobial stewardship programs is to improve patient care and health outcomes; however, much of the existing research surrounding stewardship programs has focused on promoting appropriate utilization, cost containment, and reduction in resistance rates. ${ }^{1,2}$ Development of novel ways in which antimicrobial stewardship programs can facilitate optimal infection-related patient care is needed, ${ }^{2}$ and utilization of comprehensive care bundles by antimicrobial stewardship teams have been proposed to improve care of patients. ${ }^{3}$ Care bundles are used to systematically manage specific diseases to optimize quality of care by ensuring that all aspects of clinical care are completed. ${ }^{3,4}$ Various infection-related care bundles have demonstrated improved management and outcomes of patients with sepsis, Clostridium difficile infection, and ventilatorassociated pneumonia. ${ }^{5-7}$ To our knowledge, no previously published reports have evaluated the impact of a care bundle on the management of patients with candidemia; thus, our objective was to analyze the impact of a comprehensive care bundle directed by our institution's antimicrobial stewardship team (AST) on the management of candidemia.

\section{Methods}

\section{Study Design and Setting}

This single-center, quasi-experimental study was designed to evaluate the effectiveness of a comprehensive care bundle, developed by the institution's AST, to increase compliance with activities related to the overall management of patients with candidemia. Management of candidemia was compared between the group receiving the care bundle and historical controls,

From the Departments of Pharmacy (A. Antworth, C.D. Collins, A. Kunapuli, P. Carver, K. Klein, and J.L. Nagel), and Infection Control and Hospital Epidemiology (L. Washer), University of Michigan Hospital and Health Systems, and the Division of Infectious Diseases (T. Gandhi, L. Washer), University of Michigan College of Pharmacy (A. Kunapuli, K. Klein, and P. Carver), Ann Arbor, Michigan.

Dr. Carver has received research support from Asellas Pharma US, Inc., and Merck \& Co., Inc.

For questions or comments, contact Jerod Nagel, Pharm.D., University of Michigan Hospital and Health Systems, 1500 East Medical Center Drive, UH B2D301, Ann Arbor, MI 48109; e-mail: nageljl@umich.edu. using a pretest-posttest design. Institutional review board approval was obtained for this study.

The study was conducted at the University of Michigan Health System (Ann Arbor, MI), which is a 930-licensed bed, level 1 trauma center serving both adult and pediatric patients. The trauma center reports approximately 45,000 inpatient hospital stays annually. The institution's AST was established in 1997 and is composed of four dedicated infectious disease clinical pharmacy specialists and three infectious diseases physicians. One pharmacist and one physician from the AST are dedicated to stewardship efforts in the pediatric population. In addition to daily stewardship activities, pharmacy team members provide other patient care responsibilities, and team physicians have clinic and consultation service commitments.

\section{Patient Population}

All patients with candidemia were identified using the institution's electronic medical record and clinical microbiology reports. Eligible patients included both adult and pediatric patients who had one or more positive blood cultures for Candida species during the two study periods: a historical control period from June 1, 2010-December 31, 2010, and an AST intervention period from June 1, 2011-December 31, 2011. Patients who died during the study were excluded from the primary analysis, as these patients would not have the opportunity to complete all the elements of the care bundle.

\section{Data Collection}

Data collection was accomplished by review of the electronic medical records and included information on demographics, microbiologic parameters, antifungal therapy, removal and replacement of intravascular catheters, ophthalmologic examinations, and other potential metastatic foci or complicated infections. In addition, data on the following clinical outcomes were reported: length of hospitalization, time to clearance of blood cultures, persistent blood cultures for more than 72 hours, and recurrent candidemia within 4 weeks.

\section{Historical Control Period}

During the historical control period of this study, our institution used several strategies for 
candidemia management. Clinical microbiology laboratory personnel alerted the ordering clinician to initial positive blood culture results, and further management was deferred to the patient's physician. Institutional guidelines for the management of candidemia, which were available on the hospital's internal Web site and published in an antimicrobial handbook, recommended empiric fluconazole therapy for patients with candidemia (yeast on Gram's stain), except for patients in an intensive care unit who were hemodynamically unstable or who had recently received azole therapy, an echinocandin was then recommended as empiric therapy. Deescalation to fluconazole was encouraged if the isolate demonstrated susceptibility to fluconazole, and the guidelines recommended ophthalmologic examination and removal of intravascular catheters, if possible. Patients received infectious disease consultations at the clinician's discretion.

The AST involvement in the historical group consisted of reviewing patients who were receiving antifungal agents (e.g., voriconazole, posaconazole, echinocandins, and liposomal amphotericin) which required prior authorization based on the institution's antimicrobial stewardship policy. The AST would determine if initial therapy was appropriate according to institutional criteria and followup on culture results to determine if deescalation was possible. The AST did not provide recommendations on nonpharmacologic management of comprehensive care bundle elements.

\section{Antimicrobial Stewardship Team Intervention Period}

In developing the comprehensive care bundle, the AST identified key elements from the Infectious Diseases Society of America's Clinical Practice Guidelines for the Management of Candidiasis. ${ }^{8}$ The team then recommended the following activities as part of the bundle: selection of appropriate antifungal therapy based on culture and susceptibility results; removal of intravascular catheters; repeat blood cultures at least every 48 hours until negative; appropriate duration of antifungal treatment; and ophthalmologic examination to evaluate Candida endophthalmitis.

During the AST intervention period, members of the AST used real-time surveillance and clinical-decision support software (TheraDoc, Inc., Salt Lake City, UT) to identify patients with candidemia. As in the historical control phase, the patients' physicians were notified by the micro- biology laboratory of initial positive blood culture results demonstrated yeast on Gram's stain. In addition, AST members received real-time notification of positive culture results; they were paged between 7 A.M. and 5 P.M. on Monday through Friday and e-mailed during off-hours and weekends during the intervention period. Other than the addition of the TheraDoc alerts in the AST period, there were no differences in testing or reporting of positive cultures between the two periods evaluated.

A secure, Web-based data collection application (REDCap, version 3.6.6; Biostat International, Inc., Nashville, TN) was used to track patients with candidemia during the intervention period. The database contained a checklist of bundle elements and included a free-text function allowing for communication among AST members. Patients were evaluated daily until all bundle elements were completed or until the patient died or was discharged from the hospital. Active intervention by AST members included education on candidemia management, recommendations for antifungal selection and duration of therapy, requests for repeat blood cultures, and ophthalmologic examination, as well as infectious disease consultation if there was concern for metastatic foci of infection.

Successful compliance with individual care bundle elements was defined by the AST. Appropriate antifungal therapy was defined as initiation of therapy according to institutional criteria within 72 hours of reporting antifungal susceptibility to Candida, with time being measured from susceptibility results to placement of appropriate antifungal order. Due to lack of AST coverage overnight and on weekends, the decision was made a priori to use 72 hours as the cut-off period for initiation of appropriate therapy. Fluconazole was considered appropriate therapy unless the Candida isolate exhibited a fluconazole minimum inhibitory concentration (MIC) of $32 \mu \mathrm{g} / \mathrm{ml}$ or greater; or the patient was allergic, intolerant, or had other contraindications to fluconazole. A alternative antifungal agent was considered standard of therapy if the patient had a concomitant fungal infection requiring expanded-spectrum antifungal coverage. Treatment with high-dose fluconazole (800 mg every $24 \mathrm{hrs}$ in adults, or a renally adjusted, equivalent dose) or another antifungal agent susceptible to the isolate was considered acceptable if the fluconazole MIC was revealed as susceptible-dose dependent (MIC $16 \mu \mathrm{g} / \mathrm{ml}$ ). For the bundle element of removing intravenous 
catheters, compliance was considered successful if the catheter was removed or replaced, or if the clinician documented that there was no alternative central venous access. Blood cultures had to be repeated at least every 48 hours from the time of the initial positive Gram's stain until the results were negative. The appropriate length of antifungal therapy for uncomplicated candidemia from an infected intravenous catheter was defined as 14 days after the first negative blood culture, or at least 4 weeks for documented disseminated disease. Ophthalmologic examinations were to be performed on all patients unless they were neutropenic, in which case, the examination was to be completed after resolution of neutropenia (absolute neutrophil count $>500$ cells $/ \mathrm{mm}^{3}$ ).

The AST also evaluated risk factors for potential metastatic foci of infection or complicated infection (persistently positive blood cultures for $>72 \mathrm{hrs}$, or the presence of a prosthetic heart valve, vascular graft, or implantable pacemaker or defibrillator) and, if identified, encouraged the primary care team to consider an infectious diseases consultation or additional testing to evaluate for metastatic disease.

\section{Statistical Analysis}

Statistical analysis was performed with GraphPad software (GraphPad Software, La Jolla, CA). Data were evaluated using the $\chi^{2}$ test for categoric data and $t$ tests for continuous data. Successful compliance with the individual elements of the candidemia care bundle was analyzed using the Fisher exact test. A p value of 0.05 or less was considered to indicate a statistically significant difference.

\section{Results}

Seventy-eight patients with candidemia were included in the study: 37 in the historical control group and 41 in the AST group. Patient demographic and clinical characteristics were similar between the groups (Table 1). The majority of patients in both groups were in the intensive care, solid organ transplantation, bone marrow transplantation, or hematology-oncology services. The predominant source of candidemia was from intravenous catheters, and the most commonly isolated Candida species were C. albicans, C. glabrata, and C. parapsilosis. Empiric micafungin and fluconazole therapy were similar in both groups; $31.7 \%$ and 35.1 received
Table 1. Characteristics of the Study Patients

\begin{tabular}{|c|c|c|}
\hline & $\begin{array}{c}\text { Control Group } \\
(n=37)\end{array}$ & $\begin{array}{c}\text { Antimicrobial } \\
\text { Stewardship } \\
\text { Intervention } \\
\text { Group } \\
(\mathrm{n}=41)\end{array}$ \\
\hline $\begin{array}{l}\text { Age }(\mathrm{yrs}) \\
\text { mean } \pm \mathrm{SD} \\
(\text { range })\end{array}$ & $41.3 \pm 24.3(0-91)$ & $48 \pm 22(0-85)$ \\
\hline Male & $20(54.1)$ & $17(41.5)$ \\
\hline \multicolumn{3}{|l|}{ Service } \\
\hline Adults & $30(81.1)$ & $36(87.8)$ \\
\hline Intensive care & $15(40.5)$ & $22(53.7)$ \\
\hline $\begin{array}{l}\text { Hematology- } \\
\text { oncology }\end{array}$ & $2(5.4)$ & $4(9.8)$ \\
\hline $\begin{array}{l}\text { Bone marrow } \\
\text { transplantation }\end{array}$ & $1(2.7)$ & $0(0)$ \\
\hline $\begin{array}{l}\text { Solid organ } \\
\text { transplantation }\end{array}$ & $0(0)$ & $1(2.4)$ \\
\hline Pediatrics & $7(18.9)$ & $5(12.2)$ \\
\hline Intensive care & $4(10.8)$ & $2(4.9)$ \\
\hline $\begin{array}{l}\text { Hematology- } \\
\text { oncology }\end{array}$ & $3(8.1)$ & $0(0)$ \\
\hline $\begin{array}{l}\text { Bone marrow } \\
\text { transplantation }\end{array}$ & $0(0)$ & $0(0)$ \\
\hline $\begin{array}{l}\text { Solid organ } \\
\text { transplantation }\end{array}$ & $0(0)$ & $0(0)$ \\
\hline \multicolumn{3}{|l|}{ Candida species } \\
\hline albicans & $18(48.7)$ & $17(41.5)$ \\
\hline glabrata & $7(18.9)$ & $11(26.8)$ \\
\hline parapsilosis & $3(8.1)$ & $7(17.1)$ \\
\hline tropicalis & $1(2.7)$ & $2(4.9)$ \\
\hline lusitaniae & $3(8.1)$ & $2(4.9)$ \\
\hline Other & $4(10.8)$ & $0(0)$ \\
\hline Multiple species & $1(2.7)$ & $2(4.9)$ \\
\hline \multicolumn{3}{|l|}{ Candidemia source } \\
\hline $\begin{array}{l}\text { Intravenous } \\
\text { catheter }\end{array}$ & $25(67.6)$ & $31(75.6)$ \\
\hline Intraabdominal & $4(10.8)$ & $3(7.3)$ \\
\hline Genitourinary & $3(8.1)$ & $4(9.8)$ \\
\hline Other & $5(13.5)$ & $3(7.3)$ \\
\hline \multicolumn{3}{|c|}{ Empiric antifugal therapy } \\
\hline Micafungin & $13(35.1)$ & $13(31.7)$ \\
\hline Fluconazole & $20(54.1)$ & $27(65.9)$ \\
\hline Other & $4(10.8)$ & $1(2.4)$ \\
\hline
\end{tabular}

Data are no. (\%) of patients unless otherwise specified.

${ }^{a} \mathrm{p}>0.05$ for all comparisons between the groups.

micafungin, and $65.9 \%$ and $54.1 \%$ received fluconazole in the AST and control groups, respectively. Patients with prosthetic heart valves, vascular grafts, or implantable pacemakers or cardioverter-defibrillators constituted $22 \%$ and $12 \%(p=0.4172)$ of the control and AST groups, respectively. Documented disseminated disease was not significantly different between the control and AST groups (43\% vs $27 \%, \mathrm{p}=0.15$ ), of which ophthalmologic disease was the most common site of dissemination ( $22 \%$ vs $5 \%, \mathrm{p}=0.553)$. Candida endocarditis was identified in four patients: one in the AST group and three in the control group. 
Originally, 52 and 47 patients were included in the AST and control groups, respectively; however, 11 patients $(21.2 \%)$ in the AST group and 10 patients $(21.3 \%)$ in the control group died before possible completion of all bundle elements; thus, their data were excluded from analysis. Effective antifungal therapy was started within 24 hours of Gram's stain results, for all patients except two who died; they died before the availability of Gram's stain results and antifungal therapy was never started. The median time from Gram's stain until death was 4.0 and 4.5 days $(p=0.61)$ in the AST and control groups, respectively.

Compliance with all candidemia bundle elements was significantly higher in the AST group than in the control group $(78.0 \%$ vs $40.5 \%$, $\mathrm{p}=0.0016$; Table 2). The AST group, compared with the control group, had significantly improved rates of ophthalmologic examination (97.6\% vs $75.7 \%$, $p=0.0108$ ), selection of appropriate antifungal therapy after sensitivity testing ( $100 \%$ vs $86.5 \%, p=0.0488)$, and appropriate duration of therapy $(97.6 \%$ vs $67.7 \%$, $\mathrm{p}=0.0012)$. The rates of intravenous catheter removal (95.1\% vs $86.5 \%, \mathrm{p}=0.3494)$ and repeat blood cultures at least every 48 hours until negative $(85.3 \%$ vs $78.4 \%, p=0.6118)$ were higher for the AST group, but they were not statistically significantly different.

Initiation of antifungal therapy within 24 hours of a positive Gram's stain occurred in $92.7 \%$ and $89.2 \%(\mathrm{p}=0.702)$ in the AST and control groups, respectively. There were no deaths in the patients who started antifungal therapy more than 24 hours after a positive Gram's stain. The AST intervened to begin antifungal therapy for 12 patients in the AST group, with nine of those based on real-time alerts and three based on culture review. Effective empiric therapy was started in $97.5 \%$ and $97.3 \%(p>0.99)$ in the AST and control groups, respectively. One patient in each group received fluconazole $400 \mathrm{mg} /$ day for a C. glabrata bloodstream infection with susceptible-dose dependent susceptibility to fluconazole, which was defined as inappropriate empiric therapy. All seven patients in the AST group who were candidates for deescalation were appropriately changed from micafungin to fluconazole, whereas deescalation in the control group occurred in five of 10 patients $(\mathrm{p}=0.044)$. Seven patients in the control group and one patient in the AST group continued antifungal therapy beyond the recommended duration, which resulted in 83 and 5 days of excessive antifungal therapy, respectively.

The most common reason for bundle noncompliance in the AST group was failure to obtain repeat blood cultures at least every 48 hours until clearance of candidemia, despite reminders by AST members to the primary care team in each case. Other areas of noncompliance were failing to perform an ophthalmologic examination after resolution of neutropenia and failure to remove an intravenous catheter. Time to intravenous catheter removal after Gram's stain results was similar between both groups, 2.0 days in the AST group versus 1.9 days in the control group.

The infectious disease service was consulted in $73 \%$ of patients in the AST group and $76 \%$ in the control group, and bundle compliance was similar for both groups ( $69 \%$ vs $86 \%, p=0.21$ ). Pediatric patients in the AST group had significantly improved compliance with all bundle elements compared with pediatric patients in the control group ( $100 \%$ vs $14.3 \%, \mathrm{p}=0.0152$ ); all five pediatric patients reviewed by the AST were compliant, and one of seven pediatric patients in the control group were compliant.

Table 2. Compliance with the Comprehensive Care Bundle for Candidemia

\begin{tabular}{|c|c|c|c|}
\hline & $\begin{array}{l}\text { Control } \\
\text { Group } \\
(n=37)\end{array}$ & $\begin{array}{c}\text { Antimicrobial } \\
\text { Stewardship } \\
\text { Intervention } \\
\text { Group } \\
(n=41)\end{array}$ & $\mathrm{p}$ Value \\
\hline All bundle elements successfully completed & $15(40.5)$ & $32(78.0)$ & 0.0016 \\
\hline Patients with more than 1 element not completed successfully & $9(24.3)$ & $1(2.4)$ & 0.0108 \\
\hline Appropriate therapy after culture and susceptibility results & $32(86.5)$ & $41(100)$ & 0.0488 \\
\hline Intravenous catheter removed & $32(86.5)$ & $39(95.1)$ & 0.3494 \\
\hline Blood cultures every 48 hrs until negative & $29(78.4)$ & $35(85.4)$ & 0.6118 \\
\hline Appropriate duration of therapy & $25(67.6)$ & $40(97.6)$ & 0.0012 \\
\hline Ophthalmologic examination performed & $28(75.7)$ & $40(97.6)$ & 0.0108 \\
\hline
\end{tabular}

Data are no. (\%) of patients. 
No significant differences in length of stay, time to clearance of candidemia, or the incidence of recurrent candidemia were identified (Table 3). A trend toward higher rates of persistent candidemia occurred in the control group than in the AST group $(40.5 \%$ vs $22.0 \%$, $\mathrm{p}=0.126)$. The mean duration of candidemia was relatively short in both groups (4.5 vs 5.2 days), which may be due to the majority of patients having uncomplicated intravenous catheterrelated infections. The rate of recurrent candidemia within 4 weeks of completion of therapy was $5.4 \%$ and $4.9 \%$ in the control and AST groups, respectively. One patient in the control group with recurrent candidemia, occurring 20 days after hospital discharge, did not complete two of the recommended bundle elements; the patient did not have repeat cultures documenting clearance of candidemia and received a course of treatment of only 10 days.

\section{Discussion}

Our results demonstrated that an AST-implemented comprehensive care bundle improved the overall management of patients with candidemia by facilitating improvements in both drug-therapy- and non-drug-therapy-related aspects of care. Increased compliance with indicators, such as ophthalmologic examinations and intravenous catheter removal, showed that our AST-managed care bundle optimized aspects

Table 3. Clinical Outcomes in the Study Patients

\begin{tabular}{|c|c|c|c|}
\hline & $\begin{array}{l}\text { Control } \\
\text { Group } \\
(n=37)\end{array}$ & $\begin{array}{l}\text { Antimicrobial } \\
\text { Stewardship } \\
\text { Intervention } \\
\text { Group } \\
(n=41)\end{array}$ & $\mathrm{p}$ Value \\
\hline $\begin{array}{l}\text { Length of stay } \\
\text { (days), median } \\
\text { (range) }\end{array}$ & $21(2-281)$ & $20(3-160)$ & 0.918 \\
\hline $\begin{array}{l}\text { Time to clearance } \\
\text { of candidemia } \\
\text { (days), median } \\
\text { (IQR) }\end{array}$ & $3(2-5)$ & $3(1-4)$ & 0.610 \\
\hline $\begin{array}{l}\text { No. (\%) of patients } \\
\text { with recurrent } \\
\text { candidemia } \\
\text { within } 4 \text { wks of } \\
\text { completing } \\
\text { therapy }\end{array}$ & $2(5.4)$ & $2(4.9)$ & 0.916 \\
\hline $\begin{array}{l}\text { No. }(\%) \text { of patients } \\
\text { with persistent } \\
\text { candidemia } \\
\text { lasting }>72 \mathrm{hrs}\end{array}$ & $15(40.5)$ & $9(22.0)$ & 0.126 \\
\hline
\end{tabular}

of care broader in scope than simply selection of optimal drug.

In addition, we were able to make improvements in drug-related indicators, which we suspected were already well managed. We found that several patients in the historical control group received inappropriate antifungal therapy as well as inappropriate duration of therapy. These results highlight limitations to our previous approach to candidemia management, which were passive in nature and heavily relied on educational guidelines. As stated previously, institutional guidelines during both study time periods provided empiric therapy recommendations and directed antifungal recommendations. In addition, AST members reviewed patients receiving micafungin, voriconazole, and liposomal amphotericin B as part of our prior authorization process and likely facilitated deescalation when appropriate. Care bundle implementation and the associated improvements in our tracking, communication, and documentation afforded us the opportunity to substantially improve these aspects of patient care. After bundle implementation, all patients received appropriate antifungal therapy after return of culture and susceptibility data, and only a single patient received an inappropriate duration of therapy.

The primary factor associated with reduced mortality in patients with candidemia is initiation of antifungal therapy within the first 24 hours from the time a blood culture is drawn, which is a suggested performance measure in the Infectious Diseases Society of America's candidiasis guideline. $^{8,9}$ Although we excluded patients who died from our analysis, as they may not have had the opportunity to complete all bundle elements, we evaluated the timing of initial antifungal therapy in all patients. Equal percentages ( $90 \%)$ of patients who died in both groups received antifungal therapy within 24 hours of Gram's stain, and, as expected, we were unable to detect a significant difference in mortality. There are several likely explanations. First, our historical high compliance rate showed that the real-time notifications from the microbiology laboratory directed to the primary care services was already relatively successful. Second, we acknowledge as a process limitation in our strategy, that AST members did not receive these real-time notifications on evenings or weekends, and following up on real-time e-mail alerts was not required during off hours. Rapid diagnostic tests for Candida identification, such as peptide nucleic acid fluorescence in situ hybridization (PNA FISH), were 
not used at our institution during the time of this study, which could improve the time to appropriate therapy. In one study, implementation of PNA FISH demonstrated time to effective therapy and reduced costs, but the sample size was too small to detect significant reduction in mortality in patients with candidemia. ${ }^{10}$

The quasi-experimental design of this study has several limitations. First, there was no concurrent randomized control group during the AST study period, which may have minimized the chance of maturation effects and other factors unknown to the study investigators. To attempt to account for this, both the control (before care bundle implementation) and intervention (after care bundle implementation) cohorts were studied during the same calendar time frame (June-December), which would allow a similar maturation process of medical students, residents, and fellows responsible for patient care at our large academic teaching institution. In the same way, seasonal effects should be relatively similar given the chosen time periods for the two cohorts. Although randomization is superior in methodology, it could have added an additional confounder in this study, given the monthly rotation of members of medical teams. With a randomized control group, members of the medical team would likely provide care for patients in both groups during the study period and may enforce the same bundle elements on patients not randomized to active intervention, which could ultimately affect the study results.

Another limitation to this study was the small sample size. Although we demonstrated significant improvement in bundle compliance, no significant differences were seen regarding clinical outcomes. Complications, such as recurrent candidemia, persistent candidemia, and development of disseminated candidiasis, did not occur frequently enough to detect significant differences with our sample size. It is possible that significant differences may have been seen if more patients were included in the study. Any method of documenting time to clearance of candidemia could also be considered a limitation; however, there was no significant difference seen in this area, and the method used likely had little impact on time to clearance.

To our knowledge, this is the first study describing the implementation of a comprehensive care bundle by an AST targeted at management of a specific disease state. The impact of a care bundle by an AST to improve compliance with quality indicators has been previously reported, ${ }^{4}$ and investigators reported incorporation of an antimicrobial stewardship program as part of a larger bundled strategy in the management of a C. difficile outbreak. ${ }^{1}$ The candidemia care bundle implemented by our AST used workflow process changes and innovative technology resources to improve the quality of medical care delivered in a disease state associated with high morbidity and mortality; as suggested by previous researchers. ${ }^{3}$

\section{Conclusion}

A comprehensive care bundle developed and implemented by our AST improved management of patients with candidemia at our institution. We encourage further exploration into the use of care bundles by ASTs as part of their multifaceted approach to promoting appropriate antimicrobial utilization and optimizing the management of patients with infectious diseases.

\section{References}

1. Dellit TH, Owens RC, McGowan JE, et al., for the Infectious Diseases Society of America, and the Society for Healthcare Epidemiology of America. Infectious Diseases Society of America and the Society for Healthcare Epidemiology of America guidelines for developing an institutional program to enhance antimicrobial stewardship. Clin Infect Dis 2007;44:159-77.

2. Martin CA, Armitstead JA, Mynatt RP, Hoven AD. Moving antimicrobial stewardship from restriction to facilitation. Am J Health Syst Pharm 2011;68:109-10.

3. Cooke FJ, Holmes AH. The missing care bundle: antibiotic prescribing in hospitals. Int J Antimicrob Agents 2007;30:25-9.

4. Toth NR, Chambers RM, Davis SL. Implementation of a care bundle for antimicrobial stewardship. Am J Health-Syst Pharm 2010;67:746-9.

5. Duerden BI. Contribution of a government target to controlling Clostridium difficile in the NHS in England. Anaerobe 2011;17:175-9.

6. Gao F, Melody T, Daniels DF, Giles S, Fox S. The impact of compliance with 6-hour and 24-hour sepsis bundles on hospital mortality in patients with severe sepsis: a prospective observational study. Crit Care 2005;9:R764-70.

7. Morris AC, Hay AW, Swann DG, et al. Reducing ventilatorassociated pneumonia in intensive care: impact of implementing a care bundle. Crit Care Med 2011;39:2218-24.

8. Pappas PG, Kauffman CA, Andes DA, et al. Clinical practice guidelines for the management of candidiasis: 2009 update by the Infectious Diseases Society of America. Clin Infect Dis 2009;48:503-35.

9. Garey KW, Rege M, Pai MP, et al. Time to initiation of fluconazole therapy impacts mortality in patients with candidemia: a multi-institutional study. Clin Infect Dis 2006;43:25-31.

10. Reed EE, Keating E, Bauer KA, et al. Decreasing time to effective candidemia management using an antimicrobial stewardship program (ASP) approach [abstract]. Presented at the 48th annual meeting of the Infectious Diseases Society of America, Vancouver, British Columbia, Canada, October 21, 2010.

11. Muto CA, Blank MK, Marsh JW, et al. Control of an outbreak of infection with the hypervirulent Clostridium difficile BI strain in a university hospital using a comprehensive "bundle" approach. Clin Infect Dis 2007;45:1266-73. 